Authors' reply: Balanzá-Martinez et al made a detailed review of our article ${ }^{1}$ and also provided several suggestions for future research.

Although we agree with the authors that the conclusions of our article are by no means definitive, we disagree with them on the following important issues.

First, Balanzá-Martinez et al suggest that our meta-analysis 'more likely overestimates the potential for cognitive improvement' as compared with other reviews (the authors cite as an example the review by Rund $^{2}$ ). On the contrary, we think that the comparison of results in individuals with schizophrenia with controls, which is an original feature of our review, has a sobering effect as it points out that 'practice [is] more likely than cognitive remediation to account for most of the improvements observed'. On the other hand, reviews (such as the one cited earlier) in which performances in people with schizophrenia are not compared with those of controls could mistake improvement in results for improvement in cognitive abilities.

Second, commenting on the methods we used in our metaanalysis, Balanzá-Martinez et al criticise three of our options: (a) the inclusion of studies with a test-retest duration under 1 year; (b) the inclusion of open trials (especially clozapine trials); and (c) the fact that we did not differentiate between 'patients with first-episode or chronic schizophrenia and geriatric patients' They suggest therefore that it would be better to limit the analyses to a subset of the available data assuming, without formally testing, that some of the studies' characteristics significantly influence results.

At the time we made our analysis, there were only 11 studies with more than a year test-retest interval and only 4 reported data for a control group. It was not possible to limit our analysis only to this subset of studies, let alone further exclude studies or separately analyse subgroups of studies.

Instead, we chose to include all methodologically sound studies and test the role of potentially confounding variables (including test-retest interval). By using this method, we limited the loss of important available information. It is our belief that we could not gain more insight from fewer data.

As we report in our article, only 2 out of the 17 variables tested showed a larger improvement in studies with shorter test-retest intervals. Thus, for the vast majority of variables, excluding studies with shorter test-retest intervals would lead to an unjustified loss of information.

Third, Balanzá-Martinez et al consider that their suggestions may lead to results 'more helpful for clinicians, patients and caregivers'. We think that limiting the analysis to studies with large test-retest intervals (which usually have high attrition rates) or excluding the more naturalistic, open trials would achieve just the contrary.

In conclusion, although we agree with Balanzá-Martinez et al that the conclusions of our meta-analysis are not definitive, we also consider that to improve our knowledge on the subject we need new data, not new analyses, in subsets of (the same) data.

1 Szöke A, Trandafir A, Dupont ME, Méary A, Schürhoff F, Leboyer M. Longitudinal studies of cognition in schizophrenia: meta-analysis. Br J Psychiatry 2008; 192: 248-57.

2 Rund BR. A review of longitudinal studies of cognitive functions in schizophrenia patients. Schizophr Bull 1989; 24: 425-35.

Andrei Szöke, Franck Schürhoff, Marion Leboyer, AP-HP, GHU 'ChenevierMondor', Pôle de Psychiatrie, 94000, Créteil, France; Université Paris XII, Faculte de Médecine, 9400, Créteil, and INSERM U955, Créteil, France. Email:

andrei.szoke@ach.aphp.fr

doi: 10.1192/bjp.195.1.85

\section{Neural correlates of formal thought disorder}

Horn et al investigate a very important, somewhat underexplored area of neural correlates of schizophrenic speech disturbance. Given the probability of underlying deficits in contextual integration and theory of mind, formal thought disorder yields a fertile ground for structural and functional connectivity analysis in schizophrenia. Although the use of hitherto unused techniques such as resting perfusion scan to study formal thought disorder must be lauded, the results of this preliminary study must be treated with caution for various reasons.

The composite score of the Scale for the Assessment of Thought, Language and Communication (TLC) has been used as a measure of severity of formal thought disorder. The authors have administered the scale $45 \mathrm{~min}$ before the scanning procedure for each participant. It is widely perceived that uncontrolled generation of thought is required to reliably measure formal thought disorder in schizophrenia. The TLC itself lacks a standardised practical method of eliciting such thought flow in contrast to some recently developed instruments. ${ }^{2}$ The crosssectional use of the TLC to measure formal thought disorder severity must be treated with prudence.

The authors extract components from the Positive and Negative Syndrome Scale (PANSS) using factor analysis and demonstrate that none of these components correlate with formal thought disorder severity as measured by the TLC total score. The validity of factor analysis in such a small sample is questionable and not in synchrony with available factorial structures of PANSS. ${ }^{3}$ As a result, all principal components extracted were from negative symptoms in PANSS (except the conceptual disorganisation item, which was rightly excluded from further analysis). Consequently, the results only show a lack of correlation between severity of formal thought disorder and negative symptoms as measured by PANSS. Findings from the magnetic resonance imaging may still be explained by positive symptoms alone and not by formal thought disorder. Lastly, the pervasive issue of sample size in neuroimaging studies becomes more prominent when correlation analyses are attempted in whole brain analyses.

1 Horn H, Federspiel A, Wirth M, Müller TJ, Wiest R, Wang J-J, et al. Structural and metabolic changes in language areas linked to formal thought disorder. Br J Psychiatry 2009; 194: 130-8.

2 Liddle PF, Ngan ETC, Caissie SL, Anderson CM, Bates AT, Quested DJ, et al. Thought and Language Index: an instrument for assessing thought and language in schizophrenia. Br J Psychiatry 2002; 181: 326-30.

3 Fitzgerald PB, de Castella AR, Brewer K, Filia K, Collins J, Davey P, et al. A confirmatory factor analytic evaluation of the pentagonal PANSS model. Schizophr Res 2003; 61: 97-104.

Lena Palaniyappan, Division of Psychiatry, Newcastle University, Newcastle upon Tyne NE1 4LP, UK. Email: Lena.Palaniyappan@ncl.ac.uk

doi: 10.1192/bjp.195.1.85a

Authors' reply: Palaniyappan suggests using the Thought and Language Index (TLI) instead of the TLC in order to quantify formal thought disorder. The TLI, he argues, would have the advantage over the TLC of a standardised method of eliciting thought flow. Unfortunately, the TLI was established after the start of our study, ${ }^{1}$ whereas the TLC was an established instrument that has been successfully used in numerous studies as a reliable instrument to quantify formal thought disorder. ${ }^{2}$ We agree that future studies might benefit from the application of the more standardised TLI. However, the distribution of the severity of formal thought disorder in the patient group should not change 